\title{
Digital image analysis-based scoring system for endoscopic ultrasonography is useful in predicting gastrointestinal stromal tumors
}

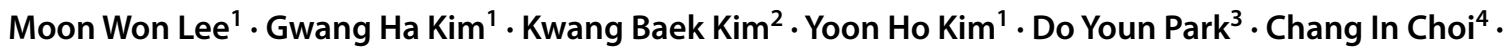 \\ Dae Hwan $\mathrm{Kim}^{4} \cdot$ Tae Yong Jeon ${ }^{4}$
}

Received: 3 October 2018 / Accepted: 18 January 2019 / Published online: 18 February 2019

(c) The International Gastric Cancer Association and The Japanese Gastric Cancer Association 2019

\begin{abstract}
Background When gastric mesenchymal tumors (GMTs) measuring $2-5 \mathrm{~cm}$ in size are found, whether to undergo further treatment or not is controversial. Endoscopic ultrasonography (EUS) is useful for the evaluation of malignant potential of GMTs, but has limitations, such as subjective interpretation of EUS images. Therefore, we aimed to develop a scoring system based on the digital image analysis of EUS images to predict gastrointestinal stromal tumors (GISTs).

Methods We included 103 patients with histopathologically proven GIST, leiomyoma or schwannoma on surgically resected specimen who underwent EUS examination between January 2007 and June 2018. After standardization of the EUS images, brightness values, including the mean $\left(T_{\text {mean }}\right)$, indicative of echogenicity, and the standard deviation $\left(T_{\mathrm{SD}}\right)$, indicative of heterogeneity, in the tumors were analyzed.

Results Age, $T_{\text {mean }}$, and $T_{\mathrm{SD}}$ were significantly higher in GISTs than in non-GISTs. The sensitivity and specificity were almost optimized for differentiating GISTs from non-GISTs when the critical values of age, $T_{\text {mean }}$, and $T_{\mathrm{SD}}$ were 57.5 years, 67.0, and 25.6, respectively. A GIST-predicting scoring system was created by assigning 3 points for $T_{\text {mean }} \geq 67,2$ points for age $\geq 58$ years, and 1 point for $T_{\mathrm{SD}} \geq 26$. When GMTs with 3 points or more were diagnosed as GISTs, the sensitivity, specificity, and accuracy of the scoring system were $86.5 \%, 75.9 \%$, and $83.5 \%$, respectively.

Conclusions The scoring system based on the information of digital image analysis is useful in predicting GISTs in case of GMTs that are $2-5 \mathrm{~cm}$ in size.
\end{abstract}

Keywords Stomach $\cdot$ Endoscopic ultrasonography $\cdot$ Gastrointestinal stromal tumor $\cdot$ Mesenchymal tumor $\cdot$ Image analysis

Electronic supplementary material The online version of this article (https://doi.org/10.1007/s10120-019-00928-w) contains supplementary material, which is available to authorized users.

Gwang Ha Kim

doc0224@pusan.ac.kr

1 Department of Internal Medicine, Pusan National University School of Medicine and Biomedical Research Institute, Pusan National University Hospital, 179 Gudeok-ro, Seo-Gu, Busan 49241, South Korea

2 Division of Computer Engineering, Silla University, Busan, South Korea

3 Department of Pathology, Pusan National University School of Medicine, Busan, South Korea

4 Department of Surgery, Pusan National University School of Medicine, Busan, South Korea

\section{Introduction}

Gastric mesenchymal tumors (GMTs) are incidentally discovered during upper endoscopy for an unrelated condition, especially during national gastric cancer screening programs in Korea, and appear as firm, protruding subepithelial tumors; however, larger tumors occasionally can cause bleeding and obstruction [1]. Histopathologically, most of these tumors are completely or partially composed of spindle cells and display smooth muscle or nerve sheath differentiation. Most GMTs are gastrointestinal stromal tumors (GISTs) derived from the interstitial cells of Cajal [1-3]. GISTs are at risk of metastatic relapse, especially in the peritoneum and liver, even after surgery for localized diseases $[4,5]$. Therefore, all GISTs are considered potentially malignant and are recommended to be resected if they are $\geq 1 \mathrm{~cm}$ in size [5-7]. 
In clinical practice, differentiation of GISTs from benign mesenchymal tumors, such as leiomyomas or schwannomas, is essential for appropriate clinical management. Endoscopic ultrasonography (EUS) is a valuable imaging tool for the evaluation of GMTs because it enables the demonstration of hypoechoic lesions that are contiguous with the fourth hypoechoic (muscularis propria) layer of the normal gastric wall [8-10]. Although there have been several studies about differentiation between GISTs and benign mesenchymal tumors using EUS $[10,11]$, the results are not consistent. Furthermore, there are limitations in the analysis of the characteristic features of such tumors due to poor interobserver agreement as a result of subjective interpretation of EUS images [12, 13].

According to recent guidelines for subepithelial tumors, when GMTs smaller than $2 \mathrm{~cm}$ are detected, they can usually be followed by periodic endoscopy or EUS once or twice a year until the tumors increase in size or become symptomatic, even if they are diagnosed as GISTs later on [14-16]. When GMTs are $2-5 \mathrm{~cm}$ in size, the decision-making process can be shared with patients regarding whether to make a histopathological diagnosis (for example, by EUS-guided fine-needle aspiration/biopsy [EUS-FNA/B]) or whether the patient should undergo further treatment [7, 15]. It is recommended that GMTs $>5 \mathrm{~cm}$ should be surgically resected and subjected to pathological examinations $[14,15]$. Therefore, if more detailed information on the malignant potential (i.e., the possibility of GIST) of GMTs measuring $2-5 \mathrm{~cm}$ can be obtained from patients' data, such as age, sex, and EUS features, these tumors can be resected endoscopically or surgically, or followed up without further invasive procedures to obtain tissue specimens, such as EUS-FNA/B or deep biopsy via endoscopic submucosal dissection [17].

Digital images consist of pixels, which are the basic elements that compose a two-dimensional picture. In digital image analysis, the distribution and spatial variation of pixels is computed using texture analysis to extract useful data, thus the digital image analysis provides objective information on EUS images, which can lessen the poor inter- and intra-observer agreements in the interpretation of EUS images. Previously, we reported the usefulness of digital image analysis of EUS images in GMTs [18]. Therefore, this study aimed to analyze differences in the clinicopathological characteristics and digital image analysis of EUS images according to the histopathology in GMTs measuring $2-5 \mathrm{~cm}$, and to develop a scoring system to predict GISTs.

\section{Methods}

\section{Subjects}

We retrospectively analyzed patients who underwent both EUS and surgical resection for GMTs such as GIST, leiomyoma, or schwannoma, at the Pusan National University Hospital (Busan, Korea) between January 2007 and June 2018. We identified 153 patients; of them, 40 patients were excluded according to the size criteria $(\leq 2 \mathrm{~cm}$ in 18 patients and $>5 \mathrm{~cm}$ in 22 patients). Thus, we included 103 patients who underwent both EUS and surgical resection for GMTs measuring $2-5 \mathrm{~cm}$. This study was reviewed and approved by the Pusan National University Hospital Institutional Review Board (IRB number, H-1808-016-070).

\section{Endoscopic ultrasonography}

EUS was performed using a radial-scanning echoendoscope (GF-UM2000; Olympus, Tokyo, Japan) at 7.5 MHz. All examinations were performed under intravenous conscious sedation (midazolam with or without propofol). The tumor was scanned after filling the stomach with $400-600 \mathrm{~mL}$ of deaerated water. At least ten still EUS images were obtained for each lesion, and these images were saved digitally in Windows bitmap format.

EUS images were reviewed by a single experienced endosonographer (GH Kim) who was blinded to the final diagnosis. Only one still EUS image of the highest quality was selected for each lesion for further digital image analysis, which was performed on a standard desktop computer.

\section{Digital image analysis}

EUS can have different brightness histograms depending on various contrasts during a real examination. As stated in our previous study [18], a standardization process was performed using the brightness values of the anechoic center and outer hyperechoic rim of the echoendoscope to minimize these differences. Image revision with histogram smoothing was also necessary to obtain better contrast because the original EUS image might be skewed by the brightness of the histogram and, therefore, might not be useful for analyzing the tumor area. Then we applied an edge linking method for all edge pixels repetitively to produce an edge. Edge linking connected and recorded all values, satisfying formula (1) in a $3 \times 3$ area to current pixel:

$\left|\nabla P\left(x_{i}, y_{i}\right)-\nabla P\left(x_{i+1}, y_{i+1}\right)\right| \leq \mathrm{Th}$

The threshold (Th) of formula (1) was set at 30, based on our preliminary study [18]. Then we applied binarization, labeling, and noise removal using morphologic information to extract the anechoic center of the echoendoscope. The outer hyperechoic rim of the echoendoscope was extracted as the area that was brighter than the neighboring pixels. Finally, formula (2) was applied to complete the standardization process: 
StandardGray $=(255-$ RimGray $) \times \frac{1+(255-\text { RimGray })}{\text { CenterGray }}$

If (CenterGray $<X<$ StandardGray), then $X=\frac{\text { StandardGray }}{\text { StandardGray }- \text { CenterGray }} \times(X-$ StandardGray $)$

Else if (StandardGray $<X<$ EdgeGray), then $X=$ StandardGray $+\frac{255-\text { StandardGray }}{\text { RimGray }- \text { StandardGray }} \times(X-$ StandardGray $)$

In formula (2), CenterGray and RimGray denote the brightness values of the anechoic center and outer hyperechoic rim of the echoendoscope, respectively; StandardGray denotes a brightness value to differentiate the anechoic center from the outer hyperechoic rim.

From the standardized image, a region of interest (ROI) was selected by an experienced endosonographer (GH Kim) for tumor analysis. The above method provides brightness information, including the minimum, maximum, mean $\left(T_{\text {mean }}\right)$, standard deviation $\left(T_{\mathrm{SD}}\right)$, median, and interquartile values (Fig. 1).

\section{Histopathology}

The tumors were immunohistochemically classified into leiomyoma, schwannoma, or GIST [3, 19]. Leiomyoma was defined as a desmin-positive and c-kit (CD117)-negative tumor, schwannoma as an S-100-positive and c-kit-negative tumor, and GIST as a c-kit, DOG-1, or CD34-positive tumor. GISTs were divided into four groups in accordance with the consensus meeting report at the National Institutes of Health [6]. According to the malignant potential of GMTs, tumors were classified largely into two groups: non-GIST (leiomyoma and schwannoma) and GIST groups.

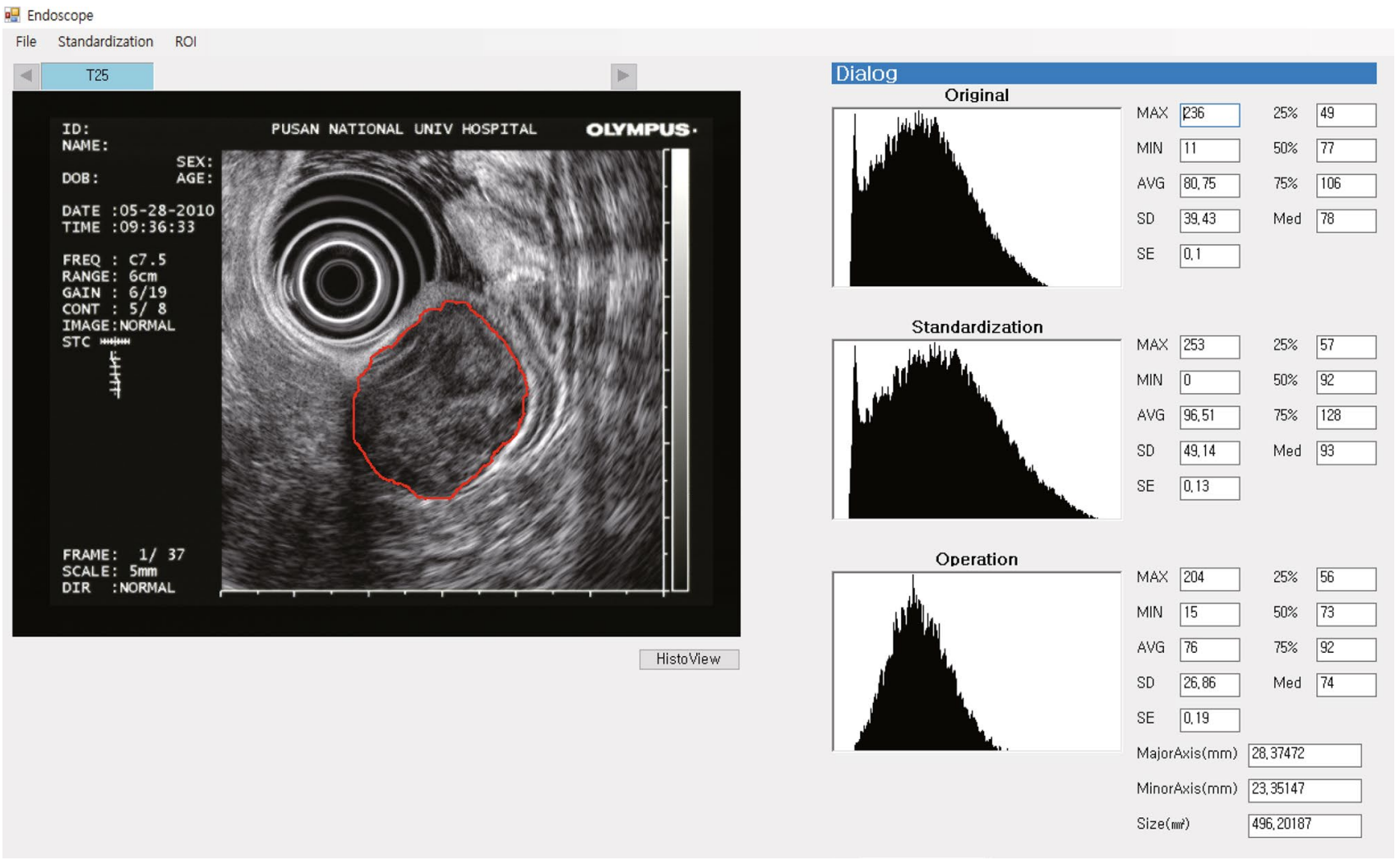

Fig. 1 An example of digital EUS image analysis. From the standardized image, a region of interest (ROI) is selected by an experienced endosonographer for tumor analysis. The final results for the ROI are expressed in the bottom histogram. The mean $\left(T_{\text {mean }}\right)$ and standard deviation $\left(T_{\mathrm{SD}}\right)$ of the brightness values are 76 and 26.86 , respectively 


\section{Statistical analysis}

Data are expressed as mean \pm SD. To identify the variables that were significantly associated with GISTs, the clinicopathological and EUS data were initially evaluated with $\chi^{2}$ test, Fisher's exact test, and Student's $t$ test. We subsequently entered the candidate variables into a logistic regression analysis. Because we attempted to make a simple and practical scoring system (GIST-predicting scoring system, GISTPSS) to distinguish GISTs from non-GISTs, clinicopathological and EUS data that were independently and statistically associated with GISTs were selected as examination items for GIST-PSS. The relative weighting of each GIST-PSS variable was based on its $\beta$-coefficient in the logistic regression analysis. Multivariate comparisons were expressed as odds ratios (OR) with $95 \%$ confidence intervals (CI). A receiver operating characteristic (ROC) curve was applied to find the best sensitivity and specificity cut-off values of age, $T_{\text {mean }}, T_{\mathrm{SD}}$, and GIST-PSS for differentiating GISTs from non-GISTs. All statistical analyses were performed using IBM SPSS version 23.0 for Windows (IBM Co., Armonk, New York, USA). Differences were considered statistically significant when $P<0.05$.

\section{Results}

\section{Clinicopathological characteristics of patients with gastric mesenchymal tumors}

The baseline clinicopathological characteristics of 103 patients who underwent EUS and surgical resection for GMTs are summarized in Table 1 . Of the 103 patients, 42 were males and 61 were females, with a mean age of 57.6 years (range $28-87$ years). Most tumors (61/103, 59.2\%) were located at the upper third of the stomach; surface ulceration was found in 25 tumors. The mean tumor size was $31.6 \mathrm{~mm}$ (range $21-50 \mathrm{~mm}$ ). The final pathological diagnoses of the tumors were leiomyoma in 15 cases, schwannoma in 14 , and GIST in 74. When the GISTs were classified according to histologic risk classification, 48 tumors were grouped as low risk, 17 as intermediate risk, and 9 as high risk.

\section{Univariate analysis for factors predicting gastrointestinal stromal tumor}

Patients in the GIST group were significantly older than those in the non-GIST group $(50.6 \pm 10.8$ years vs. $60.3 \pm 11.1$ years, $P<0.001)$. Patient sex, tumor location, and presence of surface ulceration did not differ between the two groups (Table 2). In all EUS images, the $T_{\text {mean }}$ and $T_{\mathrm{SD}}$ were calculated successfully after post-standardized image analysis. The $T_{\text {mean }}$, which is indicative of echogenicity,
Table 1 Baseline clinicopathological characteristics of 103 patients with gastric mesenchymal tumors

\begin{tabular}{ll}
\hline Characteristics & Value \\
\hline Age, years (mean \pm SD) & $57.6 \pm 11.8$ \\
Sex, $n(\%)$ & \\
Male & $42(40.8)$ \\
Female & $61(59.2)$ \\
Tumor location, $n(\%)$ & \\
Upper & $61(59.2)$ \\
Middle & $32(31.1)$ \\
Lower & $10(9.7)$ \\
Surface ulceration, $n(\%)$ & $25(24.3)$ \\
Tumor size, mm (mean \pm SD) & $31.6 \pm 7.5$ \\
Final histopathology, $n(\%)$ & \\
Leiomyoma & $15(14.6)$ \\
Schwannoma & $14(13.6)$ \\
Gastrointestinal stromal tumor & $74(71.8)$ \\
Low risk & 48 \\
Intermediate risk & 17 \\
High risk & 9 \\
\hline
\end{tabular}

Table 2 Comparison of clinicopathological and EUS characteristics between gastrointestinal stromal tumor (GIST) and non-GIST groups

\begin{tabular}{|c|c|c|c|}
\hline Characteristics & $\begin{array}{l}\text { Non-GIST } \\
\text { group } \\
(n=29)\end{array}$ & GIST group $(n=74)$ & $P$ value \\
\hline $\begin{array}{l}\text { Age, years } \\
\quad(\text { mean } \pm \mathrm{SD})\end{array}$ & $50.6 \pm 10.8$ & $60.3 \pm 11.1$ & $<0.001$ \\
\hline Sex, $n(\%)$ & & & 0.713 \\
\hline Male & $11(37.9)$ & 31 (41.9) & \\
\hline Female & $18(62.1)$ & $43(58.1)$ & \\
\hline Tumor location, $n(\%)$ & & & 0.802 \\
\hline Upper & 17 (58.6) & $44(59.5)$ & \\
\hline Middle & $10(34.5)$ & $22(29.7)$ & \\
\hline Lower & $2(8.9)$ & $8(10.8)$ & \\
\hline \multicolumn{2}{|l|}{ Surface ulceration, $n(\%)$} & & 0.120 \\
\hline Absent & $25(86.2)$ & $53(71.6)$ & \\
\hline Present & $4(13.8)$ & $21(28.4)$ & \\
\hline $\begin{array}{l}\text { Tumor size, mm } \\
(\text { mean } \pm \text { SD })\end{array}$ & $31.9 \pm 7.0$ & $31.5 \pm 7.7$ & 0.791 \\
\hline$T_{\text {mean }}($ mean $\pm \mathrm{SD})$ & $57.3 \pm 21.9$ & $88.1 \pm 25.3$ & $<0.001$ \\
\hline$T_{\mathrm{SD}}($ mean $\pm \mathrm{SD})$ & $24.5 \pm 4.7$ & $28.1 \pm 7.5$ & 0.016 \\
\hline
\end{tabular}

was significantly higher in the GIST group than in the nonGIST group $(88.1 \pm 25.3$ vs. $57.3 \pm 21.9, P<0.001)$. The $T_{\mathrm{SD}}$, which is indicative of heterogeneity, was also significantly higher in the GIST group than in the non-GIST group ( $28.1 \pm 7.5$ vs. $24.5 \pm 4.7, P=0.016)$. However, there was no significant difference in the $T_{\text {mean }}$ or $T_{\mathrm{SD}}$ between leiomyoma and schwannoma in the non-GIST group (Supplementary 
Table 1). When the GISTs were classified into benign or malignant groups according to histologic risk classification, 48 cases were grouped as benign GISTs (low risk, 48 cases) and 26 cases as malignant GISTs (intermediate risk, 17 cases; high risk, 9 cases). There was no difference in the $T_{\text {mean }}$ or $T_{\mathrm{SD}}$ between benign and malignant GISTs $(88.1 \pm 24.0$ vs. $88.0 \pm 28.0, P=0.992 ; 27.1 \pm 6.8$ vs. $30.0 \pm 8.4, P=0.106$, respectively).

An ROC curve was created to identify the best sensitivity and specificity cut-off values of age, $T_{\text {mean }}$, and $T_{\mathrm{SD}}$ for differentiating GISTs from non-GISTs (Supplementary Fig. 1). The sensitivity and specificity were almost optimized when the critical values of age, $T_{\text {mean }}$, and $T_{\mathrm{SD}}$ were 57.5 years, 67.0 , and 25.6, respectively. The sensitivity, specificity, and accuracy of age $\geq 58$ years for predicting GIST in a given tumor was $62.2 \%$ (95\% CI 55.5-67.0\%), 75.9\% (95\% CI 58.9-88.3\%), and $66.0 \%$ (95\% CI 56.5-73.0\%), respectively (Supplementary Table 2). The sensitivity, specificity, and accuracy of $T_{\text {mean }}$, and $T_{\mathrm{SD}}$ for predicting GIST were $81.1 \%$ (95\% CI 74.8-85.5\%), 79.3\% (95\% CI 63.2-90.5\%), and $80.6 \%$ (95\% CI $71.5-86.9 \%$ ), and $55.4 \%$ (95\% CI $48.7-60.9 \%), 69.0 \%$ (95\% CI 51.8-83.0\%), and 59.2\% (95\% CI $49.6-67.1 \%)$, respectively.

\section{Multivariate analysis for factors predicting gastrointestinal stromal tumor}

Multivariate logistic regression analysis revealed age $\geq 58$ years and $T_{\text {mean }} \geq 67$ were relevant independent factors predicting GIST (Table 3). The possibility of GIST in a given tumor significantly increased in patients $\geq 58$ years old (OR 6.600, 95\% CI 1.945-22.403; $P=0.002)$ and in tumors with $T_{\text {mean }} \geq 67$ (OR 16.184, 95\% CI 4.951-52.897; $P<0.001) . T_{\mathrm{SD}} \geq 26$ was associated with a possibility of GIST, but did not reach statistical significance (OR 0.947, 95\% CI 0.794-8.359; $P=0.115$ ).

\section{Establishment of gastrointestinal stromal tumor-predicting scoring system}

The GIST-PSS was created based on the above results. One point was given for $T_{\mathrm{SD}} \geq 26,2$ for age $\geq 58$ years, and 3 for $T_{\text {mean }} \geq 67$ based on the $\beta$-coefficient. Thus, the range of

Table 3 Multivariate logistic regression analysis for predicting gastrointestinal stromal tumor in gastric mesenchymal tumors

\begin{tabular}{llcr}
\hline & $\beta$-Coefficient & Odds ratio $(95 \% \mathrm{CI})$ & $P$ value \\
\hline Age $\geq 58$ years & 1.887 & $6.600(1.945-22.403)$ & 0.002 \\
$T_{\text {mean }} \geq 67$ & 2.784 & $16.184(4.951-52.897)$ & $<0.001$ \\
$T_{\mathrm{SD}} \geq 26$ & 0.947 & $2.577(0.794-8.359)$ & 0.115 \\
\hline
\end{tabular}

CI confidence interval
Table 4 Proposed gastrointestinal stromal tumor-predicting scoring system

\begin{tabular}{lll}
\hline Factors & Points & \\
\cline { 2 - 3 } & Present & Absent \\
\hline Age $\geq 58$ years & 2 & 0 \\
$T_{\text {mean }} \geq 67$ & 3 & 0 \\
$T_{\mathrm{SD}} \geq 26$ & 1 & 0 \\
\hline
\end{tabular}

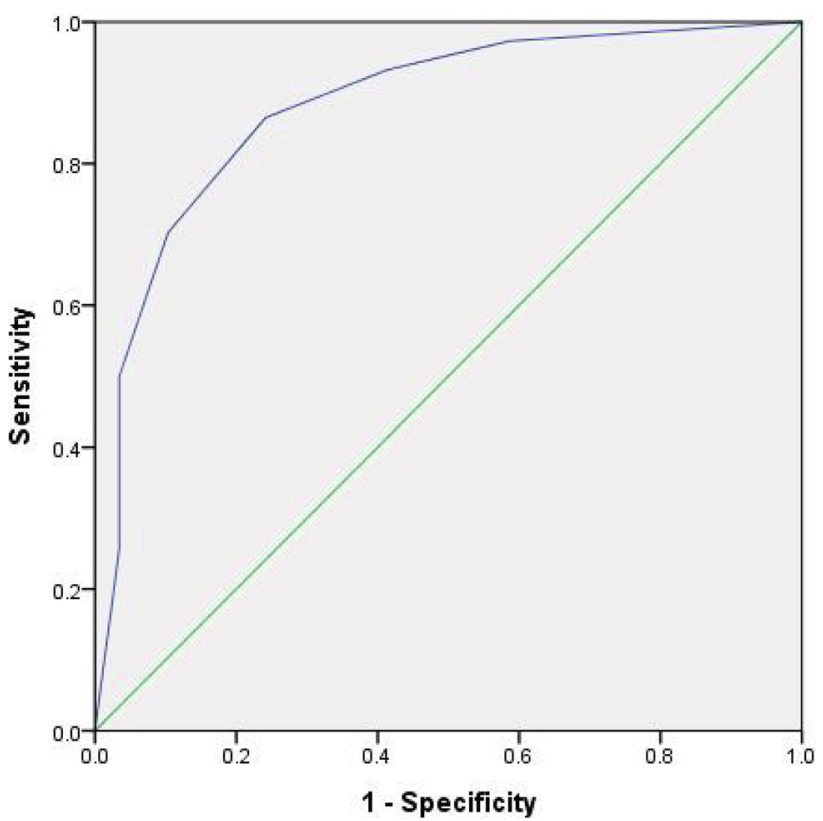

Fig. 2 Receiver operating characteristic curve used to determine cut-off values for gastrointestinal stromal tumor-predicting scoring system (GIST-PSS). The sensitivity and specificity were almost optimized when the critical values for GIST-PSS were 2.5; the respective area under the curve values was 0.861

the resulting GIST-PSS was 0-6 points (Table 4). An ROC curve was created to identify the best sensitivity and specificity cut-off values of GIST-PSS for differentiating GISTs from non-GISTs (Fig. 2). The sensitivity and specificity were almost optimized when the critical values of GIST-PSS were 2.5. Therefore, a total of three points was defined as the cutoff value between GISTs and non-GISTs. The sensitivity, specificity, and accuracy of the GIST-PSS were $86.5 \%$ (95\% CI $80.3-91.0 \%$ ), $75.9 \%$ (95\% CI 60.0-87.4\%), and $83.5 \%$ (95\% CI 74.6-90.0\%), respectively (Table 5).

\section{Discussion}

In the present study, we tried to analyze the differences in the clinicopathological characteristics and digital image analysis of EUS images according to the histopathology in GMTs 
Table 5 Sensitivity, specificity, positive and negative predictive values, and accuracy of the gastrointestinal stromal tumor (GIST)-predicting scoring system for differentiating GISTs from non-GISTs

\begin{tabular}{llllll}
\hline Predicting GIST & Sensitivity, \% (95\% CI) & Specificity, \% (95\% CI) & PPV, \% (95\% CI) & NPV, \% (95\% CI) & Accuracy, \% (95\% CI) \\
\hline Score $\geq 3$ points & $86.5(80.3-91.0)$ & $75.9(60.0-87.4)$ & $90.1(83.7-94.8)$ & $68.8(54.4-79.2)$ & $83.5(74.6-90.0)$ \\
\hline
\end{tabular}

GIST gastrointestinal stromal tumor, $P P V$ positive predictive value, $N P V$ negative predictive value, $C I$ confidence interval

measuring $2-5 \mathrm{~cm}$. We found that age $\geq 58$ years and $T_{\text {mean }}$ $\geq 67$ were independent factors predicting GIST on multivariate analysis. Additionally, the scoring system (GIST-PSS) based on age, $T_{\text {mean }}$, and $T_{\mathrm{SD}}$ showed an acceptable sensitivity, specificity and accuracy for predicting GIST in a given GMT. To the best of our knowledge, this is the first study to report a scoring system that predicts GIST using digital EUS image analysis.

We previously reported characteristic EUS findings of GISTs, schwannomas, and leiomyomas [10, 20]; heterogeneity, hyperechogenic spots, and higher echogenicity in comparison with the surrounding muscle layer were helpful for discriminating GISTs from other benign GMTs. However, interpretation of EUS images is subjective and endoscopist dependent, which results in poor interobserver agreement $[12,13]$. To overcome this limitation, we aimed to seek more objective information from EUS images.

Unlike color endoscopy images, EUS images are black and white images composed of pixels, and their echo density is expressed as brightness values from 0 to 255 . As a result, analysis of the brightness values can be an appropriate method to evaluate the level of echogenicity (expressed as $T_{\text {mean }}$ ) and the degree of heterogeneity (expressed as $T_{\mathrm{SD}}$ ) [18]. Additionally, EUS images can display different characteristics in accordance with various contrasts used during EUS examination. Therefore, to minimize these differences, we selected the brightness of the anechoic center and outer hyperechoic rim of the echoendoscope, which have the least variability, and then standardized the EUS images. We have already reported the usefulness of this standardization of EUS images in GMTs [18].

After post-standardized image analysis, both $T_{\text {mean }}$ and $T_{\mathrm{SD}}$ were significantly higher in the GIST group than in the nonGIST group on univariate analysis, which is consistent with our previous study [18]. Additionally, these findings also correspond with results of previous studies that a higher echogenicity in comparison with the surrounding muscle layer and heterogeneity are useful in diagnosing GISTs [10, 11, 21]. However, on multivariate analysis, only $T_{\text {mean }}$ was significantly higher in the GIST group than in the non-GIST group. In addition, older age ( $\geq 58$ years) was another predictor of GIST; this result is consistent with the median age of patients with GIST being 63-69 years in population-based studies $[14,22,23]$. On the contrary, the median age of patients with leiomyoma and schwannoma is $52-54$ years [10, 20, 22].
Therefore, an older age can be another objective factor for predicting GIST when a GMT is found during endoscopy.

When a subepithelial tumor is found during endoscopy, especially when one is incidentally found during routine medical health examinations, tumor size and presence or absence of clinically malignant features are the main determinants for further detailed examinations, such as EUS, computed tomography with contrast enhancement, and/or EUS-FNA/B $[14,23]$. Clinically malignant features on endoscopy include irregular borders, ulceration, and/ or growth during endoscopic follow-up. When there are no clinically malignant features, a gastric subepithelial tumor $\leq 2 \mathrm{~cm}$ is usually followed up by endoscopy or EUS once or twice a year. On the contrary, it is recommended that subepithelial tumors $>5 \mathrm{~cm}$, except for definitely benign tumors, should be surgically resected and subjected to pathological examinations $[14,15]$. In the case of gastric GMTs measuring 2-5 cm, more invasive and expensive procedures to achieve tissue diagnosis, such as EUS-FNA/B and deep biopsy via endoscopic submucosal dissection, are usually recommended to evaluate their malignant potential, mainly to differentiate GISTs from non-GISTs. Therefore, how to approach GMTs measuring $2-5 \mathrm{~cm}$ is important to both patients and physicians in real practice. We aimed to make a scoring system with an acceptable accuracy to discriminate GISTs from non-GISTs. For this, we used the factors that are not endoscopist dependent and can be expressed as objective values. In the present study, we found three objective factors (age, $T_{\text {mean }}$, and $T_{\mathrm{SD}}$ ) that were significantly associated with the possibility of GIST, and then could make GIST-PSS. When a GIST-PSS score was 3 points or more, the sensitivity, specificity, and accuracy for predicting GIST were $86.5 \%, 75.9 \%$, and $83.5 \%$, respectively. Considering the diagnostic yields of EUS-FNA/B are $60-85 \%$ in subepithelial tumors [24-26], our results are thought to be acceptable to use in real clinics. However, further large prospective studies are required to validate our results of GIST-PSS based on digital image analysis.

This study has several limitations. First, this was a retrospective study that compared EUS images between GISTs and non-GISTs using digital image analysis. Therefore, there might have been a potential bias when retrospectively reviewing the EUS images. During EUS examination, we obtained at least ten EUS images to determine the characteristics of GMTs and selected one EUS image with the 
highest quality for digital image analysis; this could compensate, to some degree, the limitation of a retrospective study. Second, patients were selected for surgery according to the clinical opinions and decisions of the physicians. Third, even though we analyzed only EUS images obtained using a radial-scanning mechanical echoendoscope (GFUM2000; Olympus) at $7.5 \mathrm{MHz}$ to reduce differences between the images due to use of different frequencies, the actual settings of the EUS, such as gain and contrast, and different echoendoscopes and EUS systems, were different in each case. Therefore, we did attempt to standardize the EUS images according to the brightness values of the anechoic center and outer hyperechoic rim of the echoendoscope. However, this attempt to standardize the EUS images cannot overcome the limitations of a retrospective study. Further prospective studies are needed that use the same settings, such as gain and contrast, during EUS examination.

In conclusion, digital EUS image analysis provides objective information to differentiate GISTs from non-GISTs in GMTs. The scoring system shows a high sensitivity and specificity in predicting GIST in GMTs measuring $2-5 \mathrm{~cm}$. Further prospective studies about the validation of the scoring system using digital image analysis are needed in a larger number of GMTs.

Acknowledgements This study was supported by Biomedical Research Institute Grant (2018B015), Pusan National University Hospital and a Busan Cancer Center Research Grant (2018), Pusan National University Hospital.

Author contributions GHK and KBK jointly conceived the study. MWL, GHK and YHK performed the data analysis. MWL, GHK, DYP, CIC, DHK, and TYJ were responsible for the data collection and contributed to the data analysis and writing of the manuscript. MWL and GHK contributed to the interpretation of the results, and reviewed the manuscript. All authors critically revised each stage of the manuscript and all authors approved the final draft for submission.

\section{Compliance with ethical standards}

Conflict of interest The authors declare that they have no competing interests.

Human rights statement All procedures followed were in accordance with the ethical standards of the responsible committee on human experimentation (institutional and national) and with the Declaration of Helsinki of 1964 and later versions.

Informed consent Informed consent or a substitute for it was obtained from all patients before they were included in the study.

\section{References}

1. Pidhorecky I, Cheney RT, Kraybill WG, Gibbs JF. Gastrointestinal stromal tumors: current diagnosis, biologic behavior, and management. Ann Surg Oncol. 2000;7:705-12.
2. Sarlomo-Rikala M, Kovatich AJ, Barusevicius A, Miettinen M. CD117: a sensitive marker for gastrointestinal stromal tumors that is more specific than CD34. Mod Pathol. 1998;11:728-34.

3. Miettinen M, Sobin LH, Sarlomo-Rikala M. Immunohistochemical spectrum of GISTs at different sites and their differential diagnosis with a reference to CD117 (KIT). Mod Pathol. 2000;13:1134-42.

4. Blay JY, Bonvalot S, Casali P, Choi H, Debiec-Richter M, Dei Tos $\mathrm{AP}$, et al. Consensus meeting for the management of gastrointestinal stromal tumors. Report of the GIST Consensus Conference of 20-21 March 2004, under the auspices of ESMO. Ann Oncol. 2005;16:566-78.

5. Miettinen M, Sobin LH, Lasota J. Gastrointestinal stromal tumors of the stomach: a clinicopathologic, immunohistochemical, and molecular genetic study of 1765 cases with long-term follow-up. Am J Surg Pathol. 2005;29:52-68.

6. Fletcher CD, Berman JJ, Corless C, Gorstein F, Lasota J, Longley BJ, et al. Diagnosis of gastrointestinal stromal tumors: a consensus approach. Hum Pathol. 2002;33:459-65.

7. Demetri GD, von Mehren M, Antonescu CR, DeMatteo RP, Ganjoo KN, Maki RG, et al. NCCN Task Force report: update on the management of patients with gastrointestinal stromal tumors. J Natl Compr Canc Netw. 2010;8(Suppl 2):1-41 (quiz S2-4).

8. Chak A, Canto MI, Rosch T, Dittler HJ, Hawes RH, Tio TL, et al. Endosonographic differentiation of benign and malignant stromal cell tumors. Gastrointest Endosc. 1997;45:468-73.

9. Palazzo L, Landi B, Cellier C, Cuillerier E, Roseau G, Barbier JP. Endosonographic features predictive of benign and malignant gastrointestinal stromal cell tumours. Gut. 2000;46:88-92.

10. Kim GH, Park do Y, Kim S, Kim DH, Choi CW, Heo J, et al. Is it possible to differentiate gastric GISTs from gastric leiomyomas by EUS? World J Gastroenterol. 2009;15:3376-81.

11. Okai T, Minamoto T, Ohtsubo K, Minato H, Kurumaya H, Oda Y, et al. Endosonographic evaluation of c-kit-positive gastrointestinal stromal tumor. Abdom Imaging. 2003;28:301-7.

12. Catalano MF, Sivak MV Jr, Bedford RA, Falk GW, van Stolk R, Presa F, et al. Observer variation and reproducibility of endoscopic ultrasonography. Gastrointest Endosc. 1995;41:115-20.

13. Gress F, Schmitt C, Savides T, Faigel DO, Catalano M, Wassef W, et al. Interobserver agreement for EUS in the evaluation and diagnosis of submucosal masses. Gastrointest Endosc. 2001;53:71-6.

14. Nishida T, Blay JY, Hirota S, Kitagawa Y, Kang YK. The standard diagnosis, treatment, and follow-up of gastrointestinal stromal tumors based on guidelines. Gastric Cancer. 2016;19:3-14.

15. Nishida T, Kawai N, Yamaguchi S, Nishida Y. Submucosal tumors: comprehensive guide for the diagnosis and therapy of gastrointestinal submucosal tumors. Dig Endosc. 2013;25:479-89.

16. Hwang JH, Rulyak SD, Kimmey MB; American Gastroenterological Association Institute. American Gastroenterological Association Institute technical review on the management of gastric subepithelial masses. Gastroenterology. 2006;130:2217-28.

17. Lee HL. Advances in the management of upper gastrointestinal subepithelial tumor: pathologic diagnosis using endoscopy without endoscopic ultrasound-guided biopsy. Clin Endosc. 2016;49:216-9.

18. Kim GH, Kim KB, Lee SH, Jeon HK, Park DY, Jeon TY, et al. Digital image analysis of endoscopic ultrasonography is helpful in diagnosing gastric mesenchymal tumors. BMC Gastroenterol. 2014;14:7.

19. Park CH, Kim GH, Lee BE, Song GA, Park DY, Choi KU, et al. Two staging systems for gastrointestinal stromal tumors in the stomach: which is better? BMC Gastroenterol. 2017;17:141.

20. Yoon JM, Kim GH, Park DY, Shin NR, Ahn S, Park CH, et al. Endosonographic features of gastric schwannoma: a single center experience. Clin Endosc. 2016;49:548-54. 
21. Williams DB, Sahai AV, Aabakken L, Penman ID, van Velse A, Webb J, et al. Endoscopic ultrasound guided fine needle aspiration biopsy: a large single centre experience. Gut. 1999;44:720-6.

22. Hu J, Liu X, Ge N, Wang S, Guo J, Wang G, et al. Role of endoscopic ultrasound and endoscopic resection for the treatment of gastric schwannoma. Medicine (Baltimore). 2017;96:e7175.

23. Yeh CN, Hwang TL, Huang CS, Lee PH, Wu CW, Chen-Guo K, et al. Clinical practice guidelines for patients with gastrointestinal stromal tumor in Taiwan. World J Surg Oncol. 2012;10:246.

24. Kim GH, Cho YK, Kim EY, Kim HK, Cho JW, Lee TH, et al. Comparison of 22-gauge aspiration needle with 22-gauge biopsy needle in endoscopic ultrasonography-guided subepithelial tumor sampling. Scand J Gastroenterol. 2014;49:347-54.
25. Han JP, Lee TH, Hong SJ, Kim HK, Noh HM, Lee YN, et al. EUSguided FNA and FNB after on-site cytological evaluation in gastric subepithelial tumors. J Dig Dis. 2016;17:582-7.

26. Zhang XC, Li QL, Yu YF, Yao LQ, Xu MD, Zhang YQ, et al. Diagnostic efficacy of endoscopic ultrasound-guided needle sampling for upper gastrointestinal subepithelial lesions: a meta-analysis. Surg Endosc. 2016;30:2431-41.

Publisher's Note Springer Nature remains neutral with regard to jurisdictional claims in published maps and institutional affiliations. 